\title{
Application of a hybrid EnKF-OI to ocean forecasting
}

\author{
F. Counillon, P. Sakov, and L. Bertino \\ Mohn Sverdrup Center/Nansen Environmental and Remote Sensing Center Thormøhlensgate 47 , Bergen, Norway \\ Received: 20 March 2009 - Published in Ocean Sci. Discuss.: 15 April 2009 \\ Revised: 7 September 2009 - Accepted: 14 September 2009 - Published: 1 October 2009
}

\begin{abstract}
Data assimilation methods often use an ensemble to represent the background error covariance. Two approaches are commonly used; a simple one with a static ensemble, or a more advanced one with a dynamic ensemble. The latter is often non-practical due to its high computational requirements. Some recent studies suggested using a hybrid covariance, which is a linear combination of the covariances represented by a static and a dynamic ensemble. Here, the use of the hybrid covariance is first extensively tested with a quasi-geostrophic model and with different analysis schemes, namely the Ensemble Kalman Filter (EnKF) and the Ensemble Square Root Filter (ESRF). The hybrid covariance ESRF (ESRF-OI) is more accurate and more stable than the hybrid covariance EnKF (EnKF-OI), but the overall conclusions are similar regardless of the analysis scheme used. The benefits of using the hybrid covariance are large compared to both the static and the dynamic methods with a small dynamic ensemble. The benefits over the dynamic methods become negligible, but remain, for large dynamic ensembles. The optimal value of the hybrid blending coefficient appears to decrease exponentially with the size of the dynamic ensemble. Finally, we consider a realistic application with the assimilation of altimetry data in a hybrid coordinate ocean model (HYCOM) for the Gulf of Mexico, during the shedding of Eddy Yankee (2006). A 10-member EnKF-OI is compared to a 10-member EnKF and a static method called the Ensemble Optimal Interpolation (EnOI). While $10 \mathrm{mem}$ bers seem insufficient for running the EnKF, the 10-member EnKF-OI reduces the forecast error compared to the EnOI, and improves the positions of the fronts.
\end{abstract}

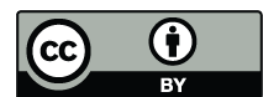

Correspondence to: F. Counillon (francois.counillon@nersc.no)

\section{Introduction}

Data assimilation methods can use ensembles to obtain and propagate the system state and the background error covariance. Two different approaches are often used. The first one, referred to as ensemble optimal interpolation (EnOI, Evensen, 2003), uses a static ensemble of model states. The second and theoretically more consistent approach, uses a dynamic ensemble, as for example the ensemble Kalman filter (EnKF, Evensen, 2007). Dynamic ensembles provide a flow-dependent background error covariance, but they can require of the order of 100 model realizations for realistic oceanic applications (Natvik and Evensen, 2003). Therefore, in practice one has to either favour the high model resolution combined with an inferior data assimilation method, or a more optimal data assimilation method at the expense of the model resolution. However, in many applications it is important to have a sufficient model resolution for obtaining a realistic representation of the dynamics. For example, Chassignet et al. (2005) show the importance of the model resolution for placing accurately the fronts in the Gulf of Mexico.

Yin and Oey (2007) and later Counillon and Bertino (2009a) investigate ensemble forecasting with a small ensemble (10 members) and a high resolution model of the Gulf of Mexico. Yin and Oey (2007) show that a probabilistic forecast provides a better accuracy than a single forecast, and Counillon and Bertino (2009a) show using an advanced perturbation system that the ensemble spread is correlated in space and time with the model error. This indicates that even small dynamic ensembles can be useful for data assimilation purposes.

Hamill and Snyder (2000) suggest a hybrid scheme called EnKF-3DVAR that combines the covariance from a dynamic ensemble with the static background covariance from 3DVAR. Each ensemble member is updated with perturbed observations. The method is tested with a quasi-geostrophic model, and it shows improvements relative to 3DVAR. The

Published by Copernicus Publications on behalf of the European Geosciences Union. 
improvements are largest in case of a sparse observation network. A similar approach was also suggested by Lorenc (2003). Etherton and Bishop (2004) suggest a hybrid scheme called the ETKF-3DVAR. It uses the ensemble transform Kalman filter (ETKF, Bishop et al., 2001) analysis scheme for updating the dynamic ensemble. In order to reduce the computational cost, only the ensemble mean is updated with the hybrid covariance, whereas the ensemble is updated using the ETKF. The ETKF-3DVAR is shown to outperform the 3DVAR in a two-dimensional turbulent model. Wang et al. (2007) use the ETKF-3DVAR, with localization for the ensemble mean, and compare it to the ESRF with localization (Whitaker and Hamill, 2002) for a two-layer primitive equation model. The ETKF-3DVAR outperforms the ESRF for small dynamic ensemble size (5 members), produces similar results for intermediate dynamic ensemble size (20 members), but is outperformed by the ESRF for larger dynamic ensembles. Finally, Wang et al. (2008a,b) demonstrates that the benefit of hybrid covariance remains when assimilating real observation on a coarse model.

For ocean applications, observations are typically less frequent than for the atmospheric applications, so that the model integration step often dominates the computational cost relative to the assimilation step. In this case, an update of both the ensemble mean and covariance using the hybrid covariance will lengthen the assimilation step, but will remain negligible with respect to the total computational cost. If using the hybrid covariance is beneficial for an update of the ensemble mean, it should also be beneficial for updating the ensemble covariance. Furthermore, an update of the ensemble mean and covariance is more in line with the Kalman Filter. We also use a method comparable to 3DVAR referred as EnOI (Evensen, 2003), which was successfully applied in the GOM (Counillon and Bertino, 2009b). To analyze the benefit of the hybrid covariance, we compare the hybrid covariance EnKF (called hereafter EnKF-OI) to the EnKF, and the hybrid covariance ESRF (called hereafter ESRF-OI) to the ESRF. The ESRF is a deterministic formulation of the EnKF, and yields a better performance than EnKF for small ensemble size (Whitaker and Hamill, 2002). Note also that another approach is proposed in Wan et al. (2009), where instead of combining the covariance matrix, the dynamic and the static ensemble are "dressed".

We see the main reason for using the hybrid covariance is expanding the subspace of ensemble anomalies produced by a small dynamic ensemble. While systems based on the ensemble Kalman filter can yield a theoretically optimal update, in practice this can only happen with an ensemble of a sufficient rank to span the system error subspace. With an ensemble of insufficient rank, the analysis becomes not only suboptimal, but also degenerative, resulting in a collapse of the ensemble. In contrast, a theoretically suboptimal EnOIbased system is often able in similar circumstances to yield meaningful updates and to provide an overall robust data assimilation system. The hybrid covariance can be therefore viewed as a compromise between a theoretically superior but computationally expensive data assimilating system based on a dynamic ensemble, and a computationally cheap and robust but theoretically inferior system based on a static ensemble.

A primary objective of our study is to test the hybrid covariance when localization and hybrid covariance are applied to both the ensemble mean and ensemble covariance, by doing a clear-cut comparison for different analysis schemes (EnKF, ESRF). A secondary objective resides in applying the hybrid covariance to a realistic application and to an ocean application.

The outline of this paper is as follows. The hybrid covariance method is presented in Sect. 2. The method is then validated on a simple 1.5-layer reduced gravity quasigeostrophic model in Sect. 3. Finally, we demonstrate the benefit of the hybrid covariance for a realistic application in the Gulf of Mexico, in Sect. 4, and present our conclusion in Sect. 5.

\section{Hybrid covariance methodology}

In sequential data assimilation, the system error covariance is often calculated from an ensemble of model states. With the EnOI, the static ensemble $A_{s}$ and the centred static ensemble $A_{s}^{\prime}$ are defined as:

$\mathbf{A}_{s}=\left[\boldsymbol{\psi}_{1}, . ., \boldsymbol{\psi}_{N_{s}}\right] \quad$ and $\quad \mathbf{A}_{s}^{\prime}=\mathbf{A}_{s}-\overline{\mathbf{A}_{s}} \in \mathcal{R}^{n \times N_{s}}$,

where $\psi$ is a model state vector, $N_{s}$ is the size of the static ensemble, $n$ is the size of the model state vector, and the overbar denotes ensemble average. The square brackets denote a horizontal concatenation of the matrices separated by a comma. A static ensemble may contain the model states sampled from a long model integration. The integration should be long enough to contain a wide variety of possible model states.

Similarly $\mathbf{A}_{d}^{\prime} \in \mathcal{R}^{n \times N_{d}}$ represents the centred dynamic ensemble matrix, where $N_{d}$ is the size of the dynamic ensemble.

The ensemble covariance matrix calculated from the static ensemble is denoted $\mathbf{C}_{s}$, and the one calculated from the dynamic ensemble $\mathbf{C}_{d}$. They are both assumed to represent the forecast error $\boldsymbol{\epsilon}$ :

$\overline{\boldsymbol{\epsilon} \boldsymbol{\epsilon}^{\mathrm{T}}} \approx \mathbf{C}_{s}=\frac{\alpha}{N_{s}-1} \mathbf{A}_{s}^{\prime}\left(\mathbf{A}_{s}^{\prime}\right)^{\mathrm{T}}$,

and

$\overline{\boldsymbol{\epsilon} \boldsymbol{\epsilon}^{\mathrm{T}}} \approx \mathbf{C}_{d}=\frac{1}{N_{d}-1} \mathbf{A}_{d}^{\prime}\left(\mathbf{A}_{d}^{\prime}\right)^{\mathrm{T}}$.

The superscript $\mathrm{T}$ denotes a matrix transpose. The variance of the static ensemble is usually different from the forecast error variance, so that a scaling factor $\alpha$ is introduced in the traditional EnOI framework (Evensen, 2003). The parameter $\alpha$ is part of the tuning of an EnOI system, and is kept constant in the following. 
As suggested by Hamill and Snyder (2000), we compute a linear combination of the two covariance matrices with an adjustable blend parameter $\beta^{1}$ :

$\widetilde{\mathbf{C}}=\beta \mathbf{C}_{s}+(1-\beta) \mathbf{C}_{d}$.

When $\beta=0$ (resp. $\beta=1$ ) the hybrid covariance is expressed entirely by the dynamic ensemble (resp. static ensemble).

Wang et al. (2007) manipulate the matrix $\widetilde{\mathbf{C}} \mathbf{H}^{\mathrm{T}} \in \mathcal{R}^{n \times m}$, with $\mathbf{H}$ being the measurement operator relating the prognostic model state variables to the measurements, and $m$ the number of measurements. However, for a large number observations $\mathbf{C} \mathbf{H}^{\mathrm{T}}$ remains a large matrix. Here the matrix $\widetilde{\mathbf{A}}^{\prime} \mathbf{H}^{\mathrm{T}} \in \mathcal{R}^{N \times m}$ is used instead, where $\widetilde{\mathbf{A}}^{\prime}$ is the matrix of combined ensemble anomalies:

$\tilde{\mathbf{A}}^{\prime}=\sqrt{N_{s}+N_{d}-1}\left[\sqrt{\frac{\beta \alpha}{N_{s}-1}} \mathbf{A}_{s}^{\prime}, \sqrt{\frac{1-\beta}{N_{d}-1}} \mathbf{A}_{d}^{\prime}\right]$,

so that

$\widetilde{\mathbf{C}}=\frac{1}{N_{s}+N_{d}-1} \widetilde{\mathbf{A}}^{\prime}\left(\widetilde{\mathbf{A}}^{\prime}\right)^{\mathrm{T}}$.

The Kalman filter equation is then solved as:

$\overline{\mathbf{A}_{d}^{a}}=\overline{\mathbf{A}_{d}^{f}}+\delta \mathbf{K}\left(\mathbf{d}-\mathbf{H} \overline{\mathbf{A}_{d}^{f}}\right)$,

$\mathbf{C}_{d}^{a}=(\mathbf{I}-\mathbf{K H}) \mathbf{C}_{d}^{f}$,

where

$\mathbf{K}=\widetilde{\mathbf{C}} \mathbf{H}^{\mathrm{T}}\left(\mathbf{H} \widetilde{\mathbf{C}} \mathbf{H}^{\mathrm{T}}+\mathbf{R}\right)^{-1}$

is the Kalman gain, $\mathbf{R}$ is the observation error covariance matrix, $\mathbf{I}$ is the identity matrix, $\mathbf{d}$ is a vector of measurements. An ensemble data assimilation system gets suboptimal due to the limited ensemble size and partially inadequate priors assumptions. Such suboptimalities can lead to an excessive reduction of the ensemble spread, which can be maintained pragmatically by multiplying the assimilation anomalies with a term $\delta$ called the ensemble inflation. The superscript "a" refers to the analysis state and "f" to the forecast.

Different schemes can be used for solving Eq. (8). In the following we apply the two most widely used: the EnKF and the ESRF. The EnKF is based on a Monte Carlo sampling of Eq. (7) and applies perturbations to the observations that can impair the stability of the results for small ensembles. To circumvent this problem, the deterministic ESRF solves the analysis Eqs. (7) and (8) without perturbing the observations, and can provide more accurate and stable results (Whitaker and Hamill, 2002).

The cost of the assimilation time step is usually in $\mathcal{O}(N \times m \times n)$. When the hybrid covariance is used,

\footnotetext{
${ }^{1} \beta$ corresponds to $1-\alpha$ in the notations of Hamill and Snyder (2000).
}

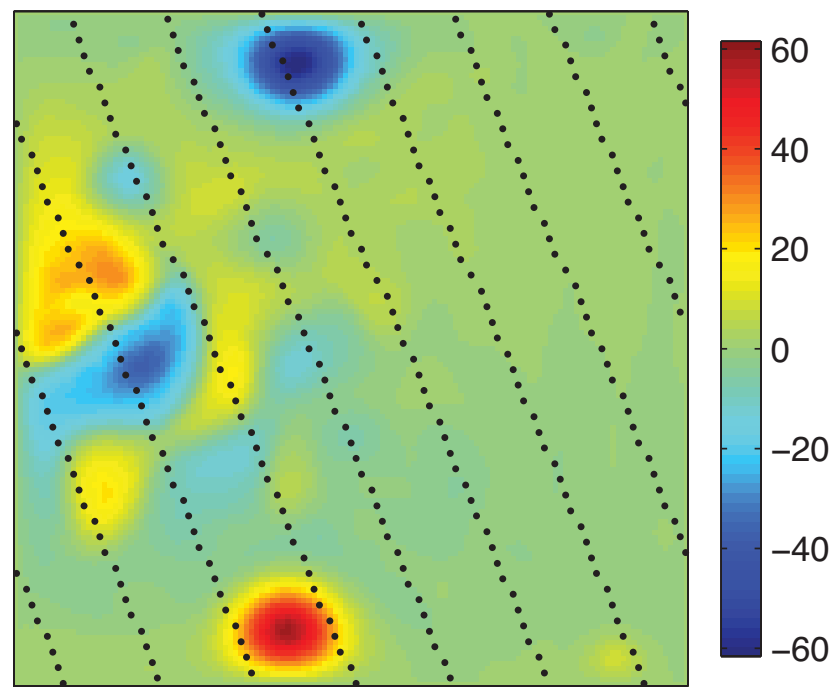

Fig. 1. Typical sea level elevation state (in dimensionless units) from the quasi-geostrophic model; the dots indicate an example of the observation locations.

$N=N_{s}+N_{d}$, and the time needed for assimilation becomes longer than both the dynamic method and the static method. However, usually $N_{d} \ll N_{s}$, so that the cost remains similar to the EnOI.

\section{A quasi-geostrophic model}

In order to analyze the capability of the hybrid covariance, we first apply it to a simple 1.5-layer reduced-gravity quasigeostrophic (QG) model with double-gyre wind forcing and biharmonic friction. It is a non-linear model with dimension $(127 \times 127)$ (see Fig. 1), and model subspace dimension of order of $10^{2}-10^{3}$. The model is eddy resolving as it generates eddies of size $\mathcal{O}(10)$ of the model grid (see Fig. 1). More details about the model are given in Sakov and Oke (2008). The model is run over 1000 model time steps, and is assimilating 300 sea surface height ( $\mathrm{SSH}$ ) observations every 10 time steps. The observations are extracted from a model run with lower viscosity, to which white noise is added with a variance ${ }^{2}$ of 4 . The observations are distributed uniformly over the domain, with a different random offset for each assimilation in order to mimic the typical distribution of satellite tracks (represented by dots on Fig. 1). Both the model code and the framework of the data assimilating system used in the experiments are available from http://enkf.nersc.no/Code/EnKF-Matlab.

\footnotetext{
${ }^{2}$ The scales and the units are dimensionless in this synthetic model.
} 


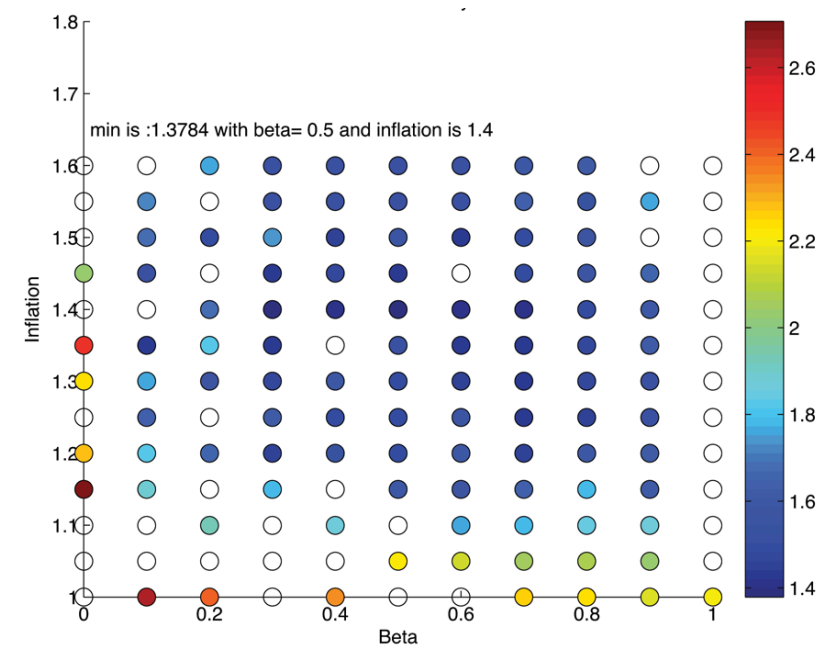

a)

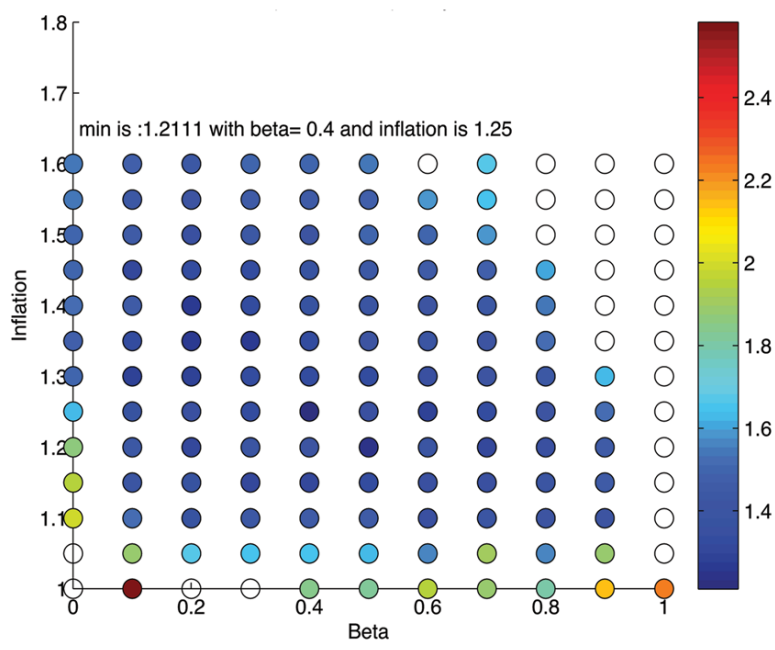

b)

Fig. 2. EnKF-OI (a) and ESRF-OI (b) values of errors $\varepsilon$ depending on the inflation $\delta$ and the blend parameter $\beta$, for 15 dynamic members and 200 static members. A white circle is used for the runs that have failed.

The parameter $\alpha$ is fixed at a value for which the EnOI is robust and performs with lowest error $^{3}(\alpha=0.04)$. This value is also optimal with the hybrid covariance method (not shown). The localization is applied with a Gaussian localization function to the state error covariance matrix by means of a Schur product (Houtekamer and Mitchell, 2001). The localization radius is set to 25 grid cells. The localization and the assimilation time step were both chosen large enough to challenge the data assimilation method. For comparison Sakov and Oke (2008) assimilate every four time steps, and show that a smaller localization radius (of approximately 15 grid cells) provides more stable and accurate results.

The hybrid covariance blends 200 static members gathered randomly over a period of 500000 model time steps with an increasing number of dynamic members until the performance in terms of RMSE saturates. Four data assimilation schemes are compared here: the EnKF, the ESRF and the hybrid covariance EnKF-OI and ESRF-OI.

The accuracy of the system is assessed by the time average root mean square error:

$$
\varepsilon=\frac{1}{p_{f}-p_{0}+1} \sum_{p=p_{0}}^{p_{f}} \sqrt{\frac{1}{n-1} \sum_{k=1}^{n}\left(\overline{\mathbf{A}_{d}^{f}}(p, k)-\boldsymbol{\psi}^{t}(p, k)\right)^{2}} .
$$

$\varepsilon$ is calculated from the ensemble mean before assimilation and start at iteration $p_{0}$ (here $p_{0}=10$ in order to remove the data assimilation spin-up time). $p_{f}$ is the total number of assimilation steps (here $p_{f}=100$ ). $\boldsymbol{\psi}^{t}$ represents a known true field, from which synthetic observations are extracted.

\footnotetext{
${ }^{3}$ Our notation of $\alpha$ follows that of Evensen (2003) and Counillon and Bertino (2009b) but corresponds to $\alpha^{2}$ in Oke et al. (2005) and in the EnKF-Matlab toolbox.
}

It is similar for all runs, and the random seed is fixed, so that all runs of similar ensemble size use the same random perturbation.

We vary the three adjustable parameters of the method, the linear blending coefficient $\beta$, the inflation parameter $\delta$ and the size of the dynamic ensemble $N_{d}$, and evaluate the resulting error $\varepsilon$. The parameter $\beta$ is chosen from 0 to 1 with an increment of 0.1 , the parameter $\delta$ from 1 to 1.6 with an increment of 0.05 , as shown in Fig. 2 where every circle represents the run average forecast error $\varepsilon$ calculated for a given $\beta, \delta$ and $N_{d}$. The values of $N_{d}$ are chosen as reported in Table 1. The optimal setting minimizes the error $\varepsilon$. The values at optimum are denoted $\varepsilon^{*}, \beta^{*}, \delta^{*}$, and are reported in Table 1.

When $\beta=0$, the EnKF-OI (resp. ESRF-OI) coincides with the EnKF (resp. ESRF) method. When $\beta=1$, every member of the dynamic ensemble is updated by the static covariance. The best guess is still provided by the ensemble average in this case, so that the method does not strictly coincide with the EnOI. In these experiments we observe that the EnKFOI and ESRF-OI with $\beta=1$ provide a slightly better estimate than the traditional EnOI, which is in agreement with the results of Wang et al. (2007). This is a consequence of the non-linearity of the QG model, because in a linear model, the integration of the ensemble mean coincides with the mean of the integrated ensemble. Note that the hybrid covariance method with $\beta=1$ is failing when inflation (or deflation) is used.

We start our experiment with 5 dynamical members. It is characterized by a big proportion of failed runs. However, there is a clear core of runs that complete for hybrid covariance methods (EnKF-OI and ESRF-OI) with high values 
Table 1. Quasi-geostrophic summary table of the $\varepsilon^{*}, \beta^{*}$, and $\delta^{*}$ obtained for each number of dynamic members with the EnKF-OI, EnKF, ESRF-OI, and ESRF analysis schemes. The EnKF-OI with a single dynamic member corresponds exactly to the EnOI. Empty cells indicate that all runs have failed for a given number of dynamic members.

\begin{tabular}{lccccccccc}
\hline$N_{d}$ & $1($ EnOI) & 5 & 10 & 15 & 20 & 25 & 30 & 35 & 40 \\
\hline EnKF-OI $\varepsilon^{*}$ & 2.32 & 1.70 & 1.45 & 1.38 & 1.29 & 1.23 & 1.22 & 1.17 & 1.15 \\
EnKF-OI $\beta^{*}$ & 1 & 0.7 & 0.6 & 0.5 & 0.6 & 0.1 & 0 & 0.4 & 0.1 \\
EnKF-OI $\delta^{*}$ & & 1.55 & 1.35 & 1.4 & 1.26 & 1.15 & 1.26 & 1.2 & 1.2 \\
EnKF $\varepsilon^{*}$ & & & & 2.03 & 1.65 & 1.31 & 1.22 & 1.20 & 1.19 \\
EnKF $\delta^{*}$ & & & & 1.45 & 1.45 & 1.15 & 1.26 & 1.2 & 1.2 \\
ESRF-OI $\varepsilon^{*}$ & 2.32 & 1.61 & 1.34 & 1.21 & 1.19 & 1.10 & 1.087 & 1.06 & 1.02 \\
ESRF-OI $\beta^{*}$ & 1 & 0.7 & 0.6 & 0.4 & 0.3 & 0.2 & 0.1 & 0.1 & 0 \\
ESRF-OI $\delta^{*}$ & 1 & 1.5 & 1.25 & 1.25 & 1.15 & 1.15 & 1.15 & 1.15 & 1.15 \\
ESRF $\varepsilon^{*}$ & & & 2.27 & 1.49 & 1.23 & 1.114 & 1.116 & 1.07 & 1.02 \\
ESRF $\delta^{*}$ & & & 1.5 & 1.35 & 1.3 & 1.15 & 1.2 & 1.15 & 1.15 \\
\hline
\end{tabular}

of $\beta$, whereas neither the EnKF nor the ESRF (i.e. with $\beta=0$ ) have completed. This indicates that the hybrid covariance methods avoid divergence in some configurations with a small dynamic ensemble, in agreement with Wang et al. (2007). Using the hybrid covariance also reduces the error $\varepsilon$ by approximately $26 \%$ compared to the EnOI. The hybrid covariance methods become more stable with 10 dynamic members, as fewer runs fail. At the same time, only a single run have complete with the ESRF, but it is of similar accuracy to the EnOI. For 15 members (see Fig. 2), all of the four schemes converge. When more than 25 dynamic members are used, the benefit from using the hybrid covariance over the EnKF/ESRF is only slight, and the best $\varepsilon^{*}$ is loosely defined on a broad range of values of $\beta$ and $\delta$.

When the dynamic ensemble is large enough, the sampling error becomes negligible. The EnOI makes the additional assumption that historical ensemble is representative of instantaneous forecasting error. Therefore, when dynamical ensemble is self-sufficient, one merely expects the adjunction of static covariance to deteriorate the results, therefore in this case $\beta^{*}=0$. In Table 1 , this occurs two times: for 30 dynamic members with the EnKF-OI and for 40 members with the ESRF-OI. The first occurrence with the EnKF-OI is likely to be the result from random variations, as $\beta^{*}$ is again positive with a larger dynamic ensemble. The second occurrence with the ESRF-OI seems more reliable because the ESRF with an ensemble of 40 members does converge to its maximum accuracy. After refining the discretization in $\beta$ and $\delta$, we obtain $\beta^{*}=0.05$. It indicates that even when the EnKF has nearly converged to its maximum accuracy, the use of hybrid covariances is still beneficial (see Fig. 3). This seems to contradict Wang et al. (2007) where the ESRF gives better result than the ETKF-OI for a large dynamic ensemble. However, their result may be caused by the difference between the ESRF with full localization and the ETKF with localization only for updating the ensemble mean.

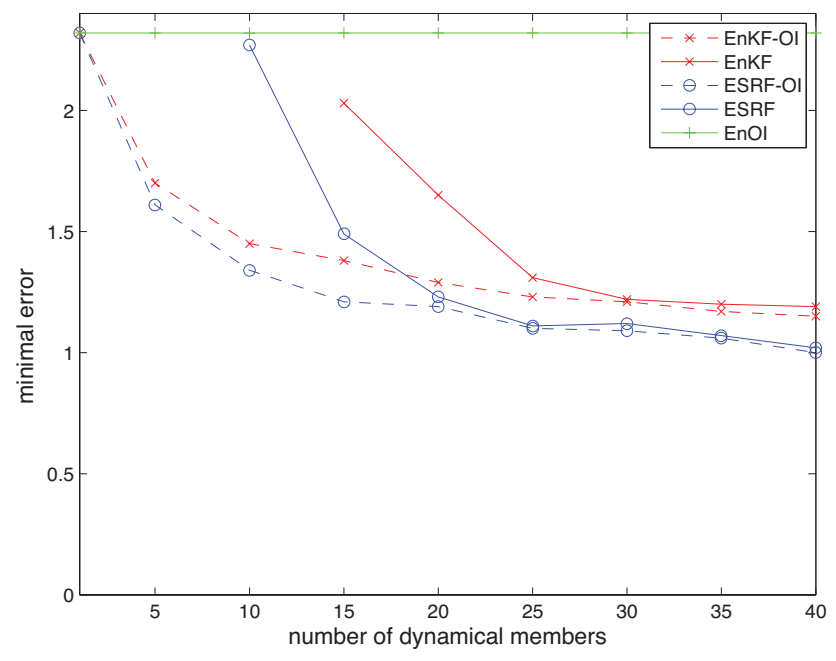

Fig. 3. Quasi-geostrophic summary plot of minimum error $\varepsilon^{*}$ vs. the dynamic ensemble size, for each data assimilation method. The observation error has standard deviation of 2 .

In Table 1 and Fig. 4 , the relationship between the blend parameter $\beta$ and the size of the dynamic ensemble $N_{d}$ is analyzed. The value of $\beta^{*}$ decreases with increasing $N_{d}$. This result seems natural, as with an increase of the dynamic ensemble size, the need for a static ensemble is reduced. In Fig. 4 , the curve of $\beta^{*}$ for the ESRF-OI is relatively regular. The relationship is noisier with the EnKF-OI due to its Monte Carlo nature, but overall the curves match, and can be fitted by an exponential (green line on Fig. 4). This indicates that the parameter $\beta^{*}$ may be independent of the analysis scheme chosen. We suggest that the value of $\beta^{*}$ reflects the balance between the rank of the dynamic ensemble and the model error subspace dimension, which implies that one can predict the optimal value of $\beta^{*}$ for a given size of dynamic ensemble, and the dimension of the model error subspace. 
Table 2. Gulf of Mexico model total average RMSE $\varepsilon_{1}$ and nowcast average RMSE $\varepsilon_{3}$ for a 10-member EnKF-OI with different values of $\beta$ and for the EnOI. The gain represents the percentage of improvement relative to the EnOI.

\begin{tabular}{lccccccccc}
\hline EnKF-OI & $\beta=0$ (EnKF) & $\beta=0.2$ & $\beta=0.5$ & $\beta=0.65$ & $\beta=0.8$ & $\beta=0.9$ & $\beta=0.95$ & $\beta=1$ & EnOI \\
\hline$\varepsilon_{1}(\mathrm{~m})$ & 0.147 & 0.075 & 0.069 & 0.07 & 0.065 & 0.066 & 0.063 & 0.067 & 0.070 \\
Total average gain $(\%)$ & -110 & -7 & 2 & 0 & 7 & 6 & 10 & 5 & 0 \\
$\varepsilon_{3}(\mathrm{~m})$ at nowcast & 0.166 & 0.094 & 0.093 & 0.094 & 0.089 & 0.088 & 0.084 & 0.09 & 0.097 \\
Nowcast gain $(\%)$ & -70 & 3 & 5 & 4 & 8 & 9 & 14 & 8 & 0 \\
\hline
\end{tabular}

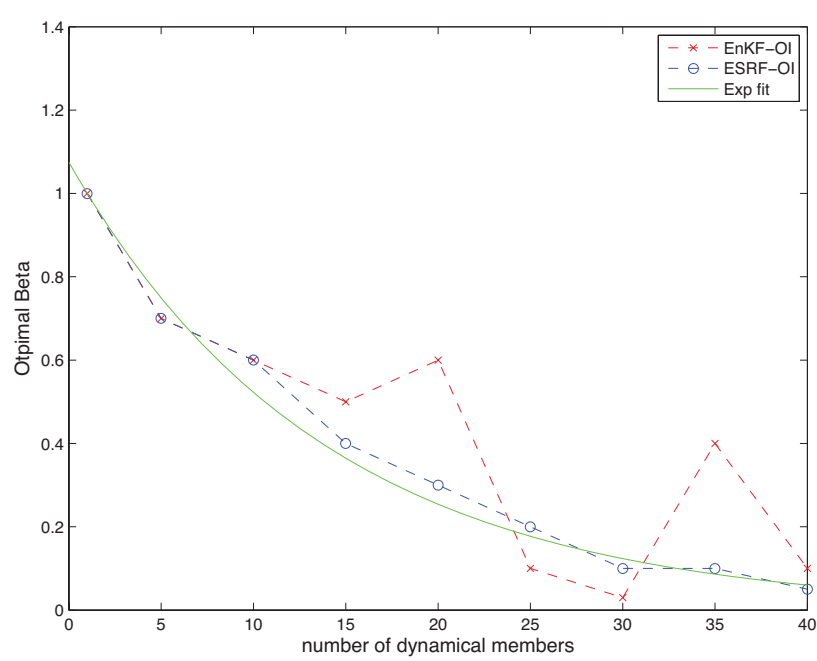

Fig. 4. Quasi-geostrophic summary plot of optimal blending coefficient $\beta^{*}$ vs. the dynamic ensemble size, for the EnKF-OI and the ESRF-OI. The green solid line is an exponential fit to the ESRF-OI experiment.

\section{A realistic application: Gulf of Mexico nested model}

The dynamics in the Gulf of Mexico (GOM) are dominated by the northward Yucatan Current flowing into a semienclosed basin. This current forms a loop (called the Loop Current, LC) and exits through the Florida Straits. At irregular intervals (Vukovich, 1988; Sturges and Leben, 2000) the LC sheds large eddies that propagate westward across the GOM. The eddy shedding involves a rapid growth of non-linear instabilities (Cherubin et al., 2005b), and these are difficult to forecast (Chassignet et al., 2005; Counillon and Bertino, 2009b). In particular, Eddy Yankee (2006) was problematic for the offshore industry operating in the northern shelf of the GOM. Thus, it is used for investigating the benefits of hybrid covariance for a realistic system conducted below.

\subsection{Experimental setup}

Chassignet et al. (2005) demonstrate the skill of HYCOM for the GOM. They emphasize the importance of both horizontal resolution and lateral boundary conditions for accurate prediction of the fronts there. A nested configuration can satisfy these two requirements with reasonable computing cost. The TOPAZ3 system provides lateral boundary conditions to a high-resolution model of the GOM (Chapter 15 in Evensen, 2007) using nesting techniques described in Browning and Kreiss (1982).

TOPAZ3 is a real-time forecasting system for the Atlantic and Arctic basins with a configuration of HYCOM, capable of monitoring the circulation patterns in the Atlantic. The grid is created using a conformal mapping of the poles to two new locations by the algorithm outlined in Bentsen et al. (1999). The horizontal resolution varies from $11 \mathrm{~km}$ in the Arctic to $18 \mathrm{~km}$ near the Equator (approximately $1 / 8^{\circ}$ ). The model is initialized from the GDEM3 climatology (Teague et al., 1990) and spin-up for 16 years. The TOPAZ3 prototype used in this work only provides the boundary conditions and does not include data assimilation, because of computer costs.

The high-resolution GOM model is set-up with $5 \mathrm{~km}$ horizontal resolution, which is sufficient to resolve the mesoscale features considering the Rossby radius in the area $\left(R_{o} \simeq 30 \mathrm{~km}\right)$. The model uses a fourth order numerical scheme for treating the advection of momentum in the primitive equations (Winther et al., 2007). To minimize the spinup time, the initial state is interpolated from an equilibrium state of TOPAZ3, and spin-up for 3 years.

For both models, there are 22 layers in the vertical. The bathymetry is specified using the General Bathymetric Chart of the Oceans database (GEBCO) with $1^{\prime}$ resolution, interpolated to the model grids. The models are forced by the 6-hourly and $0.5^{\circ}$ analyzed fields from the European Centre for Medium range Weather Forecasting (ECMWF, see http://www.ecmwf.int). The system is described in more detail in Counillon and Bertino (2009b).

At the time of writing the article, we can afford a dynamic ensemble of 10 members in real-time. Here we compare a 10-member EnKF-OI, a 10-member EnKF, and an EnOI by assimilating altimetry data. The EnOI uses a historical ensemble that is composed of 122 weekly model outputs over a 2.5 years period, without data assimilation. All methods use a fixed localization radius ${ }^{4}$ of $150 \mathrm{~km}$. A smooth transition

\footnotetext{
${ }^{4}$ This may be refined by a depth-dependent radius as in Counil-
} 
is ensured at the edges of the localization area by applying a weight function to the innovations. This function depends on the distance between the observation and the target point (Counillon and Bertino, 2009b). A value of $\alpha=0.23$ is found to be optimal for both the EnOI and the EnKF-OI set-up at the nowcast forecast horizon, and has is used in the experiments below. In order to identify the optimal value of $\beta$, we run the EnKF-OI with the values shown in Table 2.

The dynamic ensemble for the EnKF and the EnKF-OI is spun-up in order to provide realistic correlation. The initial set of restart files is randomly extracted from an a three weeks EnOI run that precedes the starting date, in order to reduce the spin-up time of the ensemble. The ensemble spread is then maintained by using perturbations and inflation over the successive assimilation cycle. In Counillon and Bertino (2009a), perturbations are applied to the atmospheric forcing, the lateral boundary conditions, and the assimilated state for the GOM model. The perturbations of the assimilated state appear to control the main position of the large features of the GOM (e.g. LC, eddies), whereas perturbations of the boundary conditions (atmospheric and lateral) control the growth of cyclonic eddies at their boundary. The same technique is used here for perturbing the boundary conditions, but the assimilated state is perturbed similarly to the EnKF; i.e. by perturbing the measurements during assimilation. The perturbations of the atmospheric fields (wind stress, air temperature) are simulated with a spectral method (Evensen, 2003), using a $50 \mathrm{~km}$ decorrelation radius, which is too small to be represented in the ECMWF data, but large enough to stimulate the ocean model. The perturbations of the lateral boundary conditions are simulated by introducing a time lag to the boundary conditions, which is set randomly at the beginning of each model run, between -37 and +37 days from the actual date. This perturbation system introduces an initial barotropic imbalance, which is damped within a day. In addition, we use an ensemble inflation of $15 \%$, which increases the ensemble spread and slightly improves the accuracy over time.

The assimilated SLA maps are provided by Ssalto/Duacs on a $1 / 3^{\circ}$ Mercator grid (Le Traon et al., 2003), with a one week delay. The standard deviation of the measurements is assumed to be constant, and it is using the average value specified by the provider in the GOM area $(3 \mathrm{~cm})$. The measurements are less accurate in the coastal area, therefore measurements are selected only in regions deeper than $300 \mathrm{~m}$, which correspond in the GOM to distances of at least $50 \mathrm{~km}$ from the coast. Accordingly, a Gaussian covariance with a decorrelation radius of $50 \mathrm{~km}$ is used for the observation error. As the SLA needs to be referred to a mean SSH, a twoyears average of TOPAZ3 SSH is interpolated to the highresolution grid. Qualitatively, it compares well with the mean dynamic topography based on satellites and in situ measurements (Rio and Hernandez, 2004).

lon and Bertino (2009b).

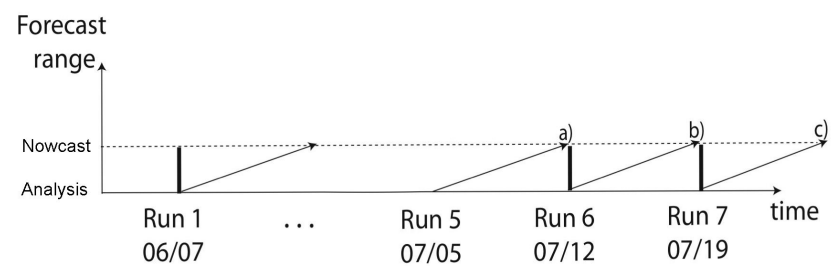

Fig. 5. Schematic of the ensemble forecast experiment. The arrows represent the model integration. The thick solid lines represent the data assimilations. The letters $(\mathbf{a}-\mathbf{c})$ correspond to the panels in Fig. 7.

The experiment starts six weeks prior to the shedding of Eddy Yankee, in order to spin-up the perturbation system (as in Counillon and Bertino, 2009a). Seven runs are processed with weekly assimilation of sea level anomaly (SLA) and are hereafter referred to as Run 1 to Run 7 (see Fig. 5). The first assimilation is applied on the 7 June and the last one on the 19 July 2006. Each run is integrated for 7 days, which corresponds to a nowcast with respect to the availability of the near real-time altimeter data.

\subsection{Forecast errors}

The benefit of the hybrid EnKF-OI over the EnOI and the EnKF is analyzed by calculating the RMSE between the model daily average SLA with the daily SLA data maps. The calculation starts from the 5 July (i.e. Run 5) to allow sufficient ensemble spin-up prior to the pre-shedding (with the non-linear growth of cyclonic eddies), the shedding and the near-reattachment of Eddy Yankee. Furthermore, this starting date corresponds to the start of available daily altimetry maps. During the shedding event, the fast dynamics of the eastern GOM contrast with the slower activity of the remaining domain. In order to clearly identify the benefit in the shedding area, the statistics are calculated over a restricted area such that the longitude is within $\left[91^{\circ} \mathrm{W} ; 83.5^{\circ} \mathrm{W}\right]$ and the latitude north of $22.25^{\circ} \mathrm{N}$. The areas shallower than $300 \mathrm{~m}$ are also excluded from the statistics due to inaccurate measurements.

In order to analyze different aspects of the performance of the data assimilation methods that use the hybrid covariance, we form the following characteristics of the system errors:

- The overall average error $\varepsilon_{1}$, for obtaining the optimal value of $\beta^{*}$ (see Table 2).

- The average of the run error $\varepsilon_{2}$, for analyzing the stability of the methods over the successive runs (see Fig. 6b).

- The error average at a given forecast horizon $\varepsilon_{3}$, for characterizing the persistence during model integration (see Fig. 6a). In particular, the error average at the nowcast is reported in Table 2. 


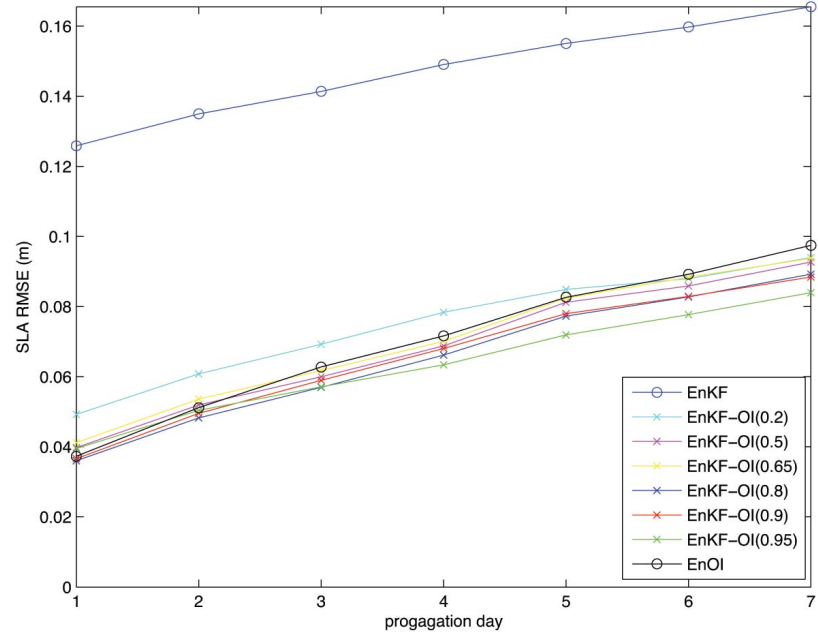

(a)

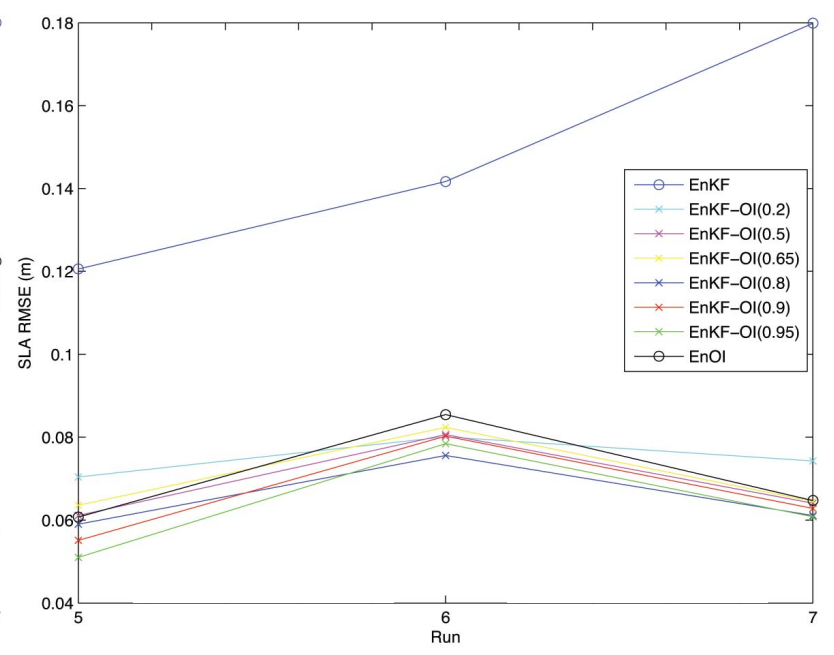

(b)

Fig. 6. Gulf of Mexico model daily error value $\left(\varepsilon_{3}\right)$ vs. forecast horizon, average over the last three runs (a). Run error average $\varepsilon_{2}$ (b).

The EnKF with 10 members yields a larger $\varepsilon_{1}$ than all the other schemes (see Table 2). This result is expected because the dynamic ensemble has too few members. In particular, we note that the error $\varepsilon_{2}$ (Fig. 6b) should decrease in the last run because the eddy has almost reattached to the LC, and the complexity of the prediction is reduced. This does not occur for the EnKF system, for which the run error average $\varepsilon_{2}$ increases with successive runs. The error for the EnKFOI with $\beta=0.2$ also does not decrease for the last run. This indicates that with 10 members, the EnKF might diverge, and that a minimum value of $\beta>0.2$ is necessary for reaching stability with the hybrid covariance method.

In Table 2, there is a clear trend indicating that high values of $\beta$ are preferable, but the minimum is once again loosely defined, possibly due to the random perturbations of observations and boundary conditions. However, the three statistics $\varepsilon_{1}, \varepsilon_{2}$, and $\varepsilon_{3}$ indicate that a value of about $\beta=0.95$ is optimal for the hybrid EnKF-OI. On average over the three runs, the EnKF-OI with $\beta=0.95$ reduces the total error average $\varepsilon_{1}$ by approximately $10 \%$, and the nowcast error average $\varepsilon_{3}$ by approximately $14 \%$ compared to the EnOI. Although the blending parameter is largely on the EnOI side, the gain in accuracy seems important.

In Fig. 6a, the error $\varepsilon_{3}$ is doubling during one week of model integration with all schemes. However, one can observe that the EnOI is diverging faster than the run with the hybrid covariance method. Counillon and Bertino (2009a) show that a high value of $\alpha$ allows for higher accuracy initially but diverges faster with model integration. A lower value of $\alpha$ tested for both the EnKF-OI and the EnOI leads to more stable but less accurate predictions at the nowcast stage.
The EnKF-OI with $\beta=1$ is performing better than the EnOI, as shown with the QG model. It is more accurate to 5\% on average, and to $8 \%$ at the nowcast stage to the EnOI (see Table 2). This indicates that imbalances caused by the perturbation system are too low to deteriorate the performance, and that the sources of variability are efficient.

\subsection{Frontal analysis}

Our main objective is to represent accurately the position of eddies (orientation, centre, shape) during the shedding events. Large velocities are located at the fronts, at the outer edge of eddies; with maximal values where the cyclonic and anticyclonic eddies interact. In this Section, we conduct a frontal analysis for the EnOI and for the EnKF-OI (with $\beta=0.95$ ) in order to qualitatively interpret the statistical gain observed above.

Chassignet et al. (2005) show that ocean colour (OC) data is useful for identifying the position of the fronts of the LC and the eddies. Furthermore, it provides an independent source of validation, and has higher resolution than the altimetry data. In Fig. 7a the deep blue contour area represents the low chlorophyll water $\left(<0.3 \mathrm{mg} / \mathrm{m}^{3}\right)$ that originates from the Caribbean Sea. The light green areas characterize the water with higher chlorophyll concentration $\left(>0.5 \mathrm{mg} / \mathrm{m}^{3}\right)$, which is usually found in areas of high biological production along the coast or within cyclonic eddies. The high chlorophyll water often propagates along the outer edge of the LC eddies, and clearly defines the front.

The position of the front is analyzed for the last three runs at the nowcast stage (represented with the letters a-c in Fig. 5) for the EnKF-OI with $\beta=0.95$ and for the EnOI. This period covers the shedding and the near reattachment of Eddy Yan- 


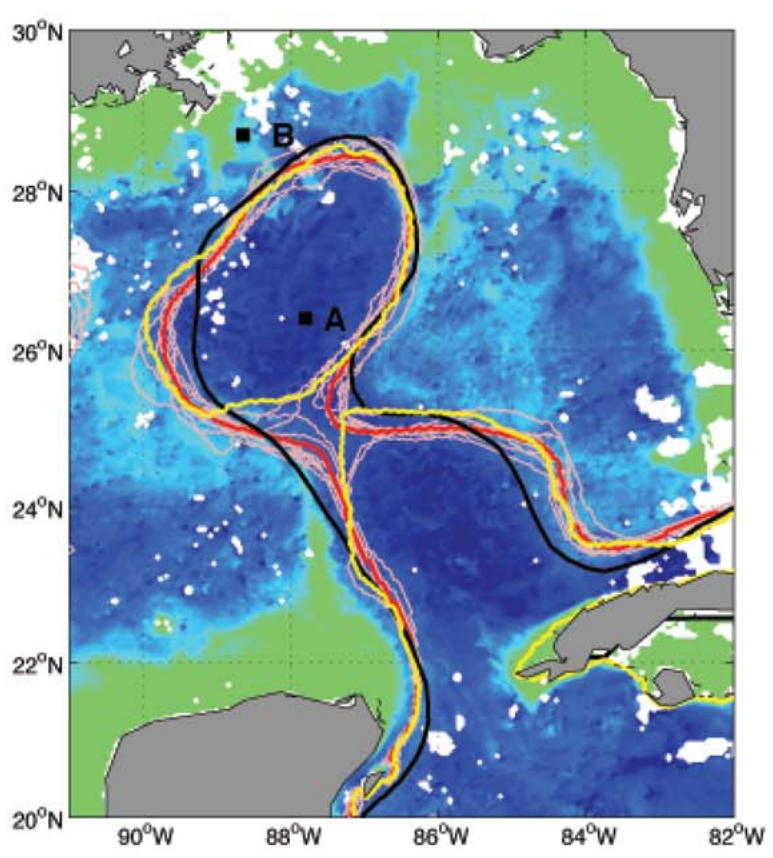

(a)

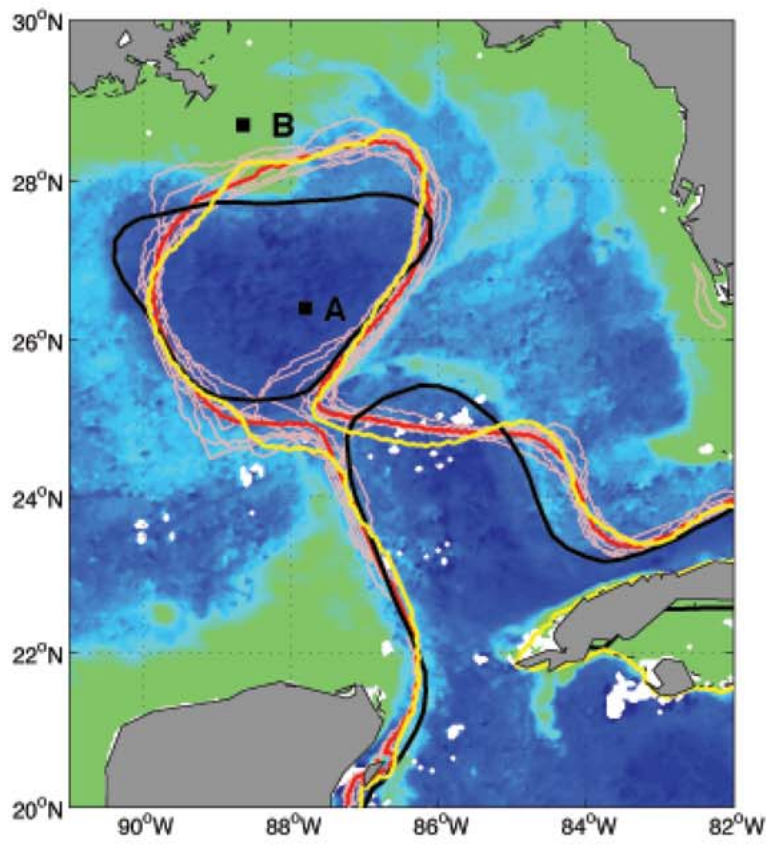

(b)

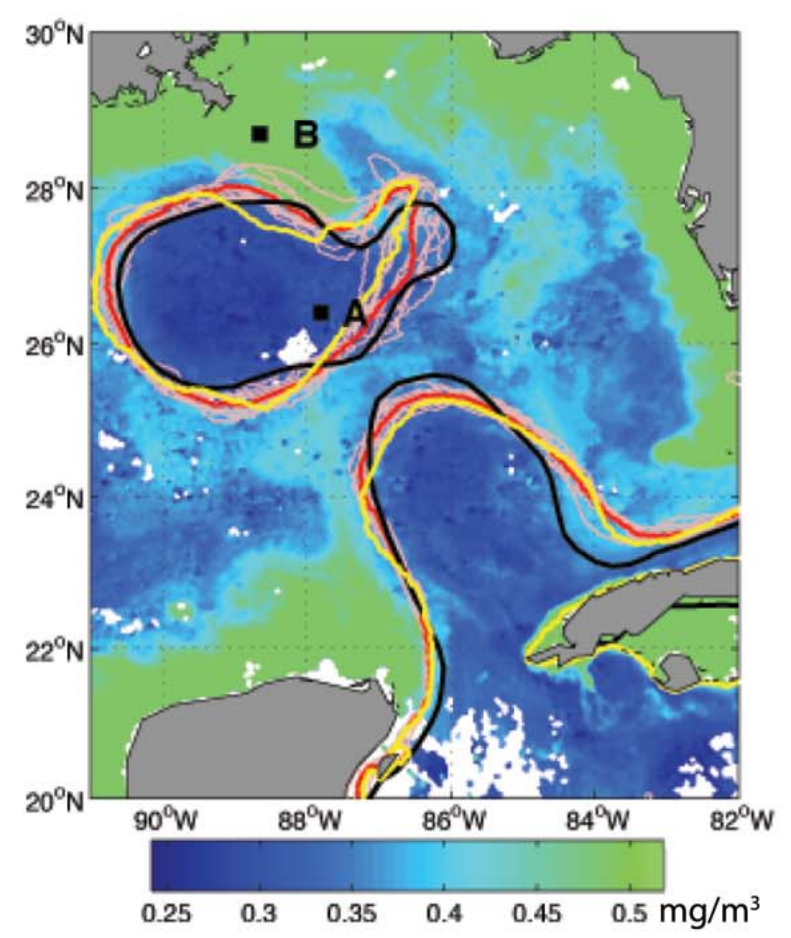

(c)

Fig. 7. Overlay of model ensemble fronts with the non-assimilated ocean colour data (contour in $\mathrm{mg} / \mathrm{m}^{3}$ ) for the $12 \mathrm{July}$ (a), the $19 \mathrm{July}$ (b), and the 26 July (c). Blue color (resp. green) indicates low (resp. high) concentration of chlorophyll, and cloud covered areas are in white. The thick black line represents the front derived from SSH altimeter data. The pink lines represent the nowcast of each ensemble member of the EnKF-OI, and the red thick line is the ensemble mean. The thick yellow line is the EnOI front. The points A and B represent the locations where correlations are analyzed in Sect. 4.4. 


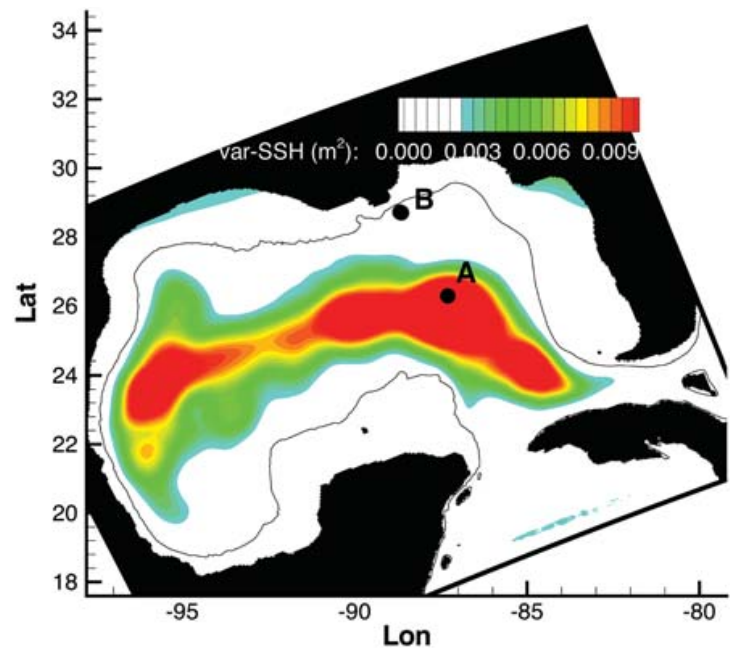

a)

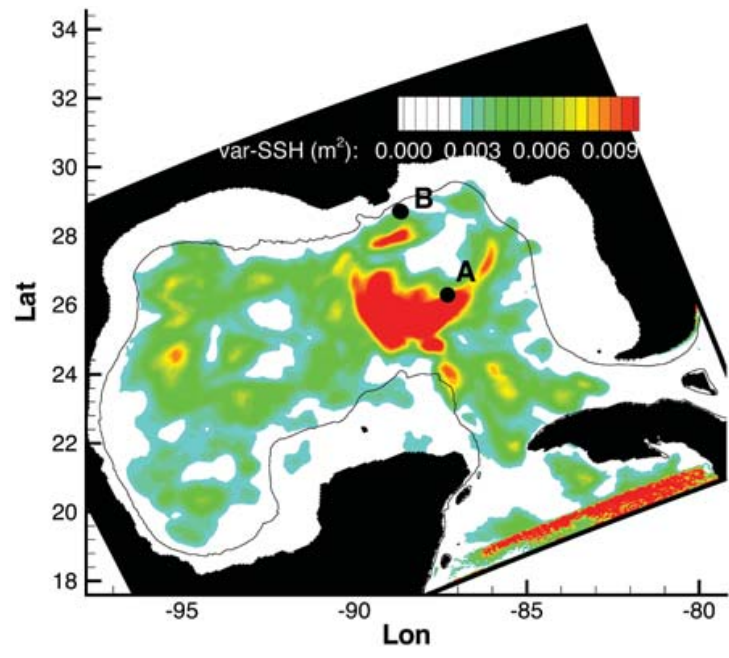

b)

Fig. 8. Ensemble variance (in $\mathrm{m}^{2}$ ) of the static (a) and of the nowcast dynamic ensemble EnKF-OI with $\beta=0.95$ on the 19 July (b). The points A and B represent the locations where correlations are analyzed in Sect. 4.4. The black line represents the $300 \mathrm{~m}$ isobaths.
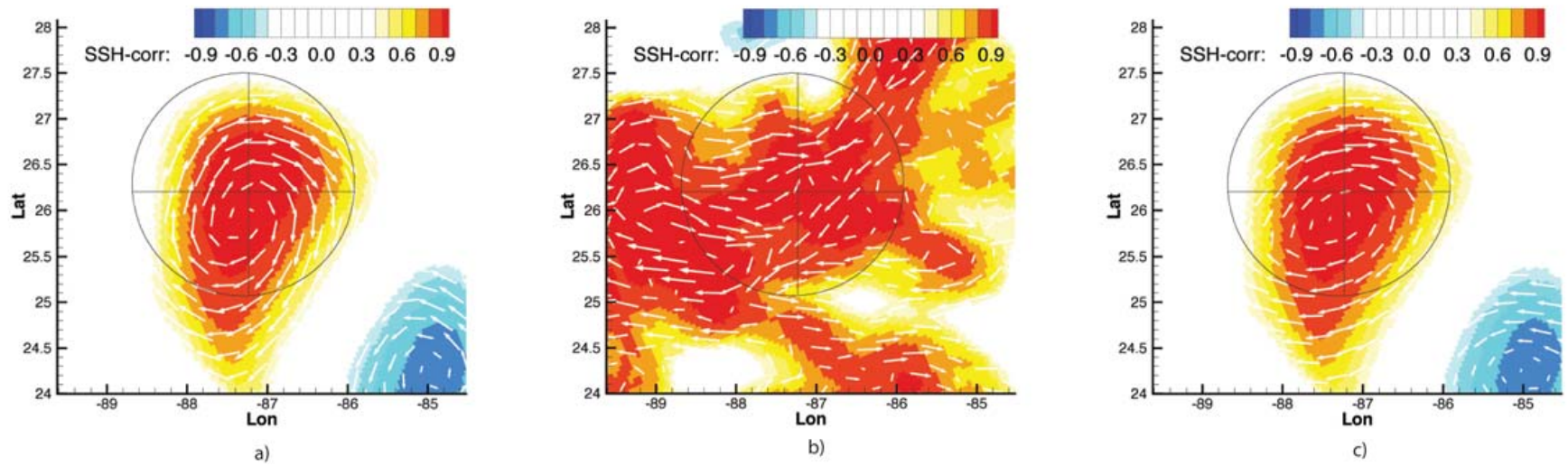

Fig. 9. Ensemble spatial correlation of SSH at the target point A (marked by a cross) for the static ensemble (a), the dynamic ensemble (b), and the hybrid ensemble (c). The white arrows represent the correlation with eastward and northward velocities. The black circle represents the localization radius.

kee (2006). On the 12 July (Fig. 7a) there is a pre-shedding scenario with a deep cyclonic penetration from the east. On the 19 July (Fig. 7b), Eddy Yankee is on the verge of being shed from the LC. On the 26 July (Fig. 7c) Eddy Yankee has shed, rotated around a cyclonic eddy located on its eastern side, and started to reattach from the east.

In Fig. 7, the ensemble fronts from the EnKF-OI are represented by pink lines over the OC data. The EnKF-OI ensemble mean provides the best guess and is represented by the thick red line. The front calculated from the EnOI is indicated by the thick yellow line. The front position derived from altimeter SSH maps (hereafter referred to as SSH data) is represented by a thick black line. Although OC data has a higher resolution, the SSH data may also be a useful indi- cator of the error in the assimilated SSH data, or be used to locate the front when clouds mask the OC data. The fronts of the model and SSH data are the $10 \mathrm{~cm} \mathrm{SSH}$ isolines, which appears to fit best with the front from OC data.

On the 12 July, the shape of the front obtained with the EnKF-OI is better positioned than that obtained with the EnOI. In particular, the eddy sheds too early with the EnOI. Furthermore, the northern front is slightly too far to the north. One can also notice that both nowcasts are relatively accurate with respect to the altimetry data.

On the 19 July, both methods misrepresent the northern front. Indeed in the OC data, the shape of Eddy Yankee is deformed by a cyclonic eddy that is squeezed between its northern front and the northern shelf. The reason is that this 


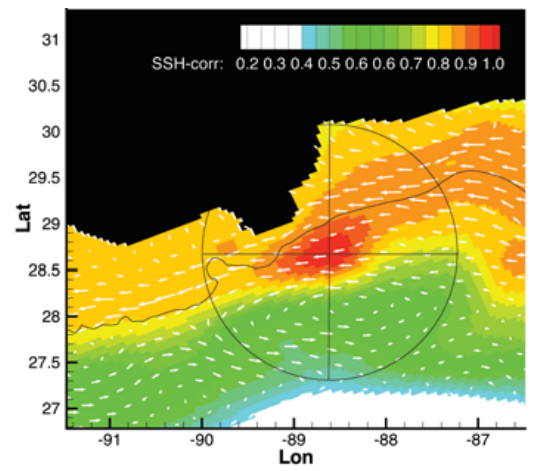

a)

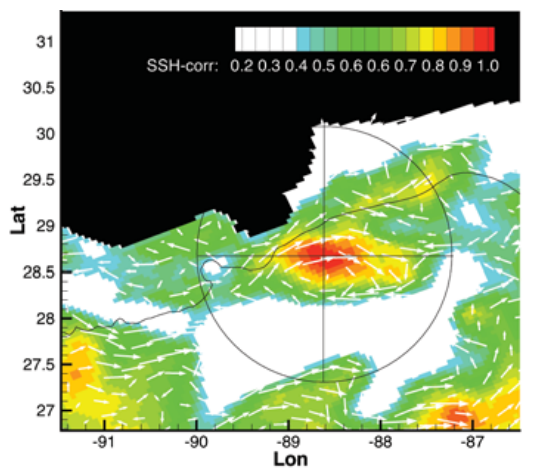

b)

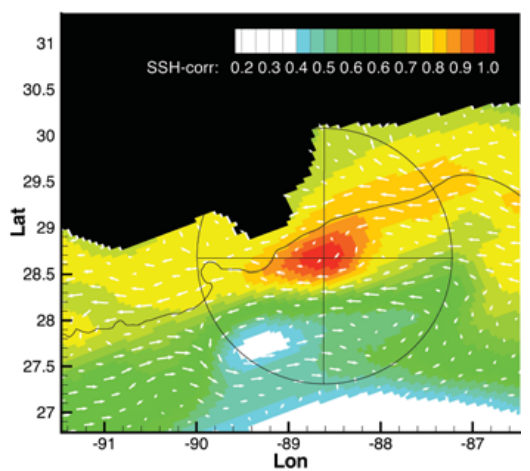

c)

Fig. 10. Ensemble spatial correlation of SSH with the target point B (marked by a cross) for the static ensemble (a), dynamic ensemble (b), and hybrid ensemble (c). The white arrows represent the correlation with eastward and northward velocities. The black circle represents the localization radius. The black line represents the $300 \mathrm{~m}$ isobath. The correlation with SSH is all positive.

cyclonic eddy was poorly represented in the gridded SLA data assimilated on the 12 July, because of its proximity to the coast. In the rest of the domain, the EnKF-OI describes the remaining connection of Eddy Yankee with the LC, whereas the eddy appears as already shed in the EnOI.

On the 26 July, the EnOI has placed Eddy Yankee slightly too far to the west, and the EnKF-OI better represent its northern front. The "comet" shape of the eddy seems to be better represented in the EnKF-OI than in the altimetry data.

One can notice that the eastern LC base is systematically too broad in the model, with both assimilation schemes. This may be caused by a model bias. Overall, the EnKF-OI improves the representation of the fronts over the EnOI during the shedding of Eddy Yankee.

\subsection{Hybrid correlation}

In order to obtain a better understanding of the benefits from the hybrid covariance, the correlation of SSH from the static, dynamic and hybrid ensembles are analyzed below. In Counillon and Bertino (2009b), a statistical analysis of the static ensemble throughout the GOM is conducted at two typical locations, one in the centre of the basin and one on the upper-shelf. The correlation is found to be realistic in the centre of the basin, but has some limitations near the uppershelf area. Here, we analyze the spatial correlation of SSH at the same locations, marked with letters A and B in Fig. 8. This analysis is repeated for the static, dynamic, and hybrid ensembles (with $\beta=0.95$ ).

Figure 8 shows the variance of the static ensemble and that of the dynamic ensemble. As expected, the variance of the static ensemble shows a maximum value induced by the resident LC base, and a positive track induced by the passage of eddies that drift westward. However, the variance at locations closer to the shelf is very small, in particular at point B. The dynamic ensemble shows a large variance near the front of Eddy Yankee, because a small displacement of a front induces a large difference in the SSH. The dynamic ensemble is expected to be most useful in the dynamic areas and in the upper-shelf coastal area, but its contribution in the middle of the basin should be small because the variance from the static ensemble dominates there.

In the centre of the basin (point A in Fig. 8), the correlation of SSH and velocity from the static ensemble clearly reflects the geostrophy, as a higher SSH increases the anticyclonic currents around the target point (see Fig. 9), and there is a slight anisotropy due to the interaction with the resident LC. The SSH correlation from the dynamic ensemble highlights the position of the southern front of the eddy, but it is noisy and uneasy to interpret. In total, the hybrid correlation is nearly identical to the static ensemble.

In the coastal area, (point B in Fig. 8), the correlation of $\mathrm{SSH}$ and velocity from the static ensemble is dominated by the seasonal variability. The problem of seasonal variability when using a static ensemble is rather common, and can be tackled e.g. by filtering out the seasonal signal (Oke et al., 2005), but this was not applied here because seasonal variability in the GOM is not large. An increase of SSH at the target point increases the SSH almost uniformly in the whole area, and induces a weak shelf current (see Fig. 10). In the dynamic ensemble, there is a positive correlation with a small anticyclonic ring, and with a vortex of opposite sign at its boundary. This correlation seems realistic because small anticyclones and cyclones with a radius smaller than $75 \mathrm{~km}$ interact with the shelf there (Hamilton and Lee, 2005), but it is noisy due to a small number of dynamic members. In the hybrid ensemble, the correlation with the anticyclonic ring is clearer than in the static ensemble, both for the SSH and currents. Furthermore, the hybrid correlation is smoother than that of the dynamic ensemble.

The benefits of using hybrid covariance compared to the static covariance may not appear as large, but they are still 
important as they occur at locations where the static covariance is known to be inadequate.

\section{Conclusions}

This study follows the work from Hamill and Snyder (2000); Lorenc (2003); Etherton and Bishop (2004); Wang et al. (2007, 2008a,b) and analyzes the benefits of using the hybrid covariance. The hybrid covariance combines the covariance from a dynamical ensemble and from a static ensemble. Unlike previous works, the hybrid covariance and localization are applied to updates both the ensemble mean and the covariance. We also evaluate the hybrid covariance with the EnKF and the ESRF analysis schemes instead of the ETKF (Etherton and Bishop, 2004; Wang et al., 2007), or of a variational approach (Hamill and Snyder, 2000). As a consequence, we found the hybrid covariance to be beneficial compared to the dynamical approach; even when a large dynamical ensemble is used. In addition, the hybrid covariance methods are applied for the first time to a realistic application (i.e. assimilating real observations, using a state of the art model at a resolution capable of resolving the dynamics, and thus applicable in an operational setting), and to the ocean.

The results with the QG model indicate that:

- Using the square-root scheme improves the stability and the accuracy compared to the EnKF. However, the conclusions regarding the hybrid covariance are independent of the analysis scheme used.

- The hybrid covariance method provides better results than both the static ensemble and the dynamic ensemble methods at equal dynamic ensemble size. The benefits are large for a small dynamic ensemble, and small for a large dynamic ensemble.

- The hybrid covariance allows a smooth transition between the EnOI and the EnKF (or ESRF).

- The optimal linear blending coefficient $\beta^{*}$ decreases exponentially with the size of the dynamic ensemble. In other words, more weight is being put towards the dynamical ensemble when its size increases.

In a realistic HYCOM configuration of the GOM, $10 \mathrm{dy}-$ namic members are insufficient for the EnKF to converge. The hybrid covariance EnKF-OI improves both the SSH RMSE (by 14\%) and the position/shape of the fronts compared to the EnOI at the nowcast stage. Using a comparison with ocean colour, we show that the improvements are related to a better representation of the fronts. However, the overall results are not as encouraging as with the QG model. It is clear, considering the very high value of $\beta$, that a further increase of the number of members should be largely beneficial. In two characteristic locations studied, the correlation calculated from a hybrid ensemble seems more realistic than that of the static and of the dynamic ensembles, and it is possible that an increase of the localization radius can further improve the accuracy of the results. Furthermore, Hamill and Snyder (2000) indicated a larger benefit when a sparse observation network data were assimilated. It seems probable that the benefit from the hybrid covariance could increase if SLA track data were assimilated instead of the interpolated maps.

The results obtained from both models indicate that the hybrid covariance methods can improve both the accuracy and the stability of the system. For 10 members, the optimal value $\beta^{*}$ is larger with the GOM model than with the QG model, which possibly indicates that the GOM model has more degrees of freedom. It could be interesting to extend the study for an increasing number of dynamic members for the GOM application when more computing power is available. It would allow for a better comparison between the QG and the GOM experiments. In particular, it would be interesting to analyze whether an exponential decrease of $\beta^{*}$ with the number of dynamic members also occurs in the GOM. This could provide a useful indication of the expected error when setting up an operational forecasting system.

Acknowledgements. We thank Jiang Zhu and Peter Oke for reviews that led to an improved version of the paper. The authors are supported by the Research Council of Norway through the project 166568 "Ocean weather and Ecosystems", 1788894 "eVITA-EnKF", and partial funding from the EU MERSEA project (SIP3-CT-2003-502885), and a grant of CPU time from the Norwegian Supercomputing Project (NOTUR).

Edited by: D. Stevens

\section{References}

Bentsen, M., Evensen, G. Drange, H. and Jenkins, A. D.: Coordinate transformation on a sphere using conformal mapping, Mon. Weather Rev., 127, 2733-2740, 1999.

Bishop, C. H., Etherton, B. J., and Majumdar, S. J.: Adaptive sampling with the ensemble transform Kalman filter. Part I: Theoretical aspects, Mon. Weather Rev., 129, 420-436, 2001.

Browning, G. L. and Kreiss, H. O.: Initialization of the shallow water equations with open boundaries by the bounded derivative method, Tellus, 34, 334-351, 1982.

Chassignet, E. P., Hulburt, H. E., Smedstad, O. M., Barron, C. N., Ko, D. S., Rhodes, R. C., Shriver, J. F., Wallcraft, A. J. and Arnone, A. R.: Assessment of data assimilative ocean models in the Gulf of Mexico using Ocean Color, Circulation in the Gulf of Mexico: Observations and Models, American Geophysical Union, 161, 87-100, 2005.

Cherubin, L. M., Morel, Y. , and Chassignet, E. P.: The role of cyclones and topography in the Loop Current ring shedding, J. Phys. Oceanogr., 31, 569-591, 2005b.

Counillon, F. and Bertino, L.: High resolution ensemble forecasting for the Gulf of Mexico eddies and fronts, Ocean Dynam., 59(1), 83-95, 2009a. 
Counillon, F. and Bertino, L.: Ensemble Optimal Interpolation: multivariate properties in the Gulf of Mexico, Tellus A, 61(2), 296-308, 2009b.

Etherton, B. J. and Bishop, C. H.: Resilience of hybrid ensemble/3DVAR analysis schemes to model error and ensemble covariance error, Mon. Weather Rev., 132, 1065-1080, 2004.

Evensen, G.: The ensemble Kalman filter: Theoretical formulation and practical implementation, Ocean Dynam., 53, 343-367, 2003.

Evensen, G.: Data assimilation: The ensemble Kalman filter, Springer-Verlag New York, Inc. Secaucus, NJ, USA, 2007.

Hamill, T. M. and Snyder, C.: A hybrid ensemble Kalman filter3D variational analysis scheme, Mon. Weather Rev., 128, 29052919, 2000.

Hamilton, P. and Lee, T. N.: Eddies and jets over the slope of the northeast Gulf of Mexico, Geophysical monograph, 161, 123142,2005

Houtekamer, P. and Mitchell, H. : A sequential ensemble Kalman filter for atmospheric data assimilation, Mon. Weather Rev., 129, 123-137, 2001.

Lorenc, A. C: The potential of ensemble Kalman filter for NWPa comparison with 4D-Var, Q. J. Meteor. Soc., 129, 3183-3203, 2003.

Natvik, L. J. and Evensen, G.: Assimilation of ocean colour data into a biochemical model of the North Atlantic Part 2. Statistical analysis, J. Marin. Syst., 40, 155-169, 2003.

Oke, P. R., Schiller, A., Griffin, D. A., and Brassington, G. B.: Ensemble data assimilation for an eddy-resolving ocean model of the Australian region, Q. J. Roy. Meteor. Soc., 131, 3301-3311, 2005.

Rio, M. and Hernandez, F.: A mean dynamic topography computed over the world ocean from altimetry, in situ measurements, and a geoid model, J. Geophys. Res., 109, 1-19, 2004.

Sakov, P. and Oke, P. R.: A deterministic formulation of the ensemble Kalman filter: an alternative to ensemble square root filters, Tellus A, 60, 361-371, 2008.
Sturges, W. and Leben, R.: Frequency of ring separations from the loop current in the Gulf of Mexico: a revisited estimate., J. Phys. Oceanogr., 30, 1814-1819, 2000.

Teague, W. J., Carron., M. J., and Hogan, P. J.: A comparision between the Generalized Digital Environmental Model and Levitus climatologies, J. Geophys. Res., 95, 7167-7183, 1990.

Le Traon, P. Y., Dibarboure, G., and Dorandeu, J.: SSALTO/DUACS and operational altimetry, Geoscience and Remote Sensing Symposium, 2003, IGARSS'03, Proceedings, 2003 IEEE International, 2, 2003.

Vukovich, F. M.: Loop Current boundary variation, J. Geophys. Res., 93, 585-15, 1988.

Wan, L., Zhu, J., and Bertino, L.: A Dressed ensemble Kalman filter using the hybrid coordinate ocean model in the Pacific, Adv. Atmos. Sci., 26(5), 1042-1052, 2009.

Wang, X., Hamill, T. M., Whitaker, J. S., and Bishop, C. H.: A comparison of hybrid ensemble transform Kalman filter-OI and ensemble square-root filter analysis schemes, Mon. Weather Rev. 135, 1055-1076, 2007.

Wang, X., Barker, D. M., Snyder, C., and Hamill, T. M.: A hybrid ETKF-3DVAR data assimilation scheme for the WRF model. Part I: Observing system Simulation Experiment, Mon. Weather Rev, 136, 5116-5131, 2008.

Wang, X., Barker, D. M., Snyder, C., and Hamill, T. M.: A hybrid ETKF-3DVAR data assimilation scheme for the WRF model Part II: Real observation experiments, Mon. Weather Rev., 136, 5132-5147, 2008.

Whitaker, J. S., and Hamill, T. M.: Ensemble data assimilation without perturbed observations, Mon. Weather Rev., 130, 19131924, 2002.

Winther, N., Morel, Y., and Evensen, G.: Efficiency of high order numerical schemes for momentum advection, J. Marin. Syst., 67, 31-46, 2007.

Yin, X. and Oey, L.: Bred-ensemble ocean forecast of loop current and rings, Ocean Model., 17, 300-326, 2007. 